\title{
A propos d'une Eccrinale parasite de Coléoptère Passalide du Laos
}

\author{
Par Jehanne-Françoise MANIER et Jean THÉODORIDÈS
}

\section{Historique des Eccrinales de Coléoptères}

Leidy signale en décembre 1849, aux Etats-Unis, la présence d'un endophyte parasite de l'intestin postérieur du Coléoptère Passalide, Passalus cornutus Fabricius. Il le nomme Enterobrus attenuatus, le genre Enterobrus ayant été créé par lui deux mois plus tôt (1849 a) pour le Thallophyte du proctodeum d'un Myriapode Diplopode. Leidy (1850) modifie le terme d'Enterobrus en Enterobryus qui sera désormais adopté ; l'année 1853, il complète l'étude de Enterobryus attenuatus. Plus de cinquante ans se sont écoulés quand Léger et Duboscq (1905), en France, évoquent le cas d'Eccrinales parasites de Coléoptères aquatiques; ces auteurs ne décrivent pas les endophytes, mais notent que leurs caractères spéciaux nécessiteront la création de nouveaux genres. Léger et Duboscq (1916) étudient à nouveau les Eccrinales d'Hydrophilides et créent deux espèces qu'ils rattachent provisoirement au genre Eccrinopsis, établi par eux en 1906 pour des Thallophytes d'Isopodes; ils jugent alors utile de placer, dans le genre Eccrinopsis, Enterobryus attenuatus de Leidy qui devient Eccrinopsis attenuatus (Leidy). Thaxter (1920) décrit, fixé à l'air libre sur les plaques anales d'un Passalus sp. des Antilles: Enterobryus compressus. Léger et Duboscq (1929) remanient encore la nomenclature des Eccrinales de Coléoptères et proposent le genre Trichella pour les endophytes d'Insectes. Ils notent cependant qu'ils n'ont pas observé, dans ce genre, les spores durables, éléments fondamentaux de discrimination. Poisson (1931) étudie deux nouvelles espèces hébergées par des Hydrophiles récoltées en France, qu'il rapporte au genre Trichella de Léger et Duboscq. Heymons (R. et H.) (1934) étudient un Enterobryus de Passalus. En France, pour les Eccrinales de larves de Scarabaeidae, sont créés les genres Paratrichella (Manier 1947) et Lajassiella (Tuzet et Manier 1950). Maessen (1955), au cours d'un travail d'ensemble sur les Eccrinales d'Allemagne, signale la présence de champignons filamenteux dans l'intestin postérieur de treize Hydrophilidae, un Scarabaeidae, un Staphylinidae. Elle groupe les espèces étudiées dans le genre Trichella et dans les deux nouveaux genres Lactella et Microtrichella. Des Eccrinales sont identifiées sans être nommées chez deux Scarabaeidae, un Tenebrionidae récoltés en France et un Passalidae de Madagascar (Théodoridès 1955) chez un Tenebrionidae du Congo ex-belge (Manier et 
Théodoridès, 1957). Lichtwardt (1957) donne une longue description d'Enterobryus attenuatus retrouvé dans le proctodeum de Passalus cornutus provenant de différentes régions d'Amérique.

\section{Etude de l'Eccrinale de LEPTAULAX DENTATUS F. (1)}

Six Passalidae: Leptaulax dentatus F., provenant des environs de Vientiane (Laos) et récoltés en février 1964, ayant été mis à notre disposition, quatre d'entre eux ont été sacrifiés en vue de l'étude de leurs endophytes intestinaux, deux ont été réservés à leur détermination.

L'examen des quatre Leptaulax révèle, dans tous les cas, la présence d'Eccrinales dans une courte portion de l'intestin postérieur. Le proctodeum de ce Coléoptère, comme celui des Passalides en général, est fortement contourné et se divise en trois parties: un iléon très rétréci par rapport au mésentéron délimité en avant par les tubes de Malpighi, en arrière par un caecum rempli d'Oxyurides du genre Artigasia, un côlon plus large et lobé, un rectum musculeux et étroit. Les Eccrinales, fixées à la cuticule chitineuse interne garnie d'aspérités épineuses, sont toujours localisées dans la région de transition entre l'iléon et le côlon. Elles mêlent leurs filaments à ceux beaucoup plus grêles et ramifiés d'un Schizophyte du genre Cladophytum Leidy (1849).

Les plus jeunes organismes sont constitués par la spore génératrice $(s)$, une courte zone de prolifération (p), un organe de fixation $(f)$ (fig. I, 1). Dans des conditions favorables, la zone de prolifération s'allonge rapidement, ses noyaux se multiplient (fig. I, 2); les filaments végétatifs bien développés peuvent mesurer $2 \mathrm{~mm}$ de long, ils ne présentent jamais de ramifications, ils sont flexueux non spiralés, leur cytoplasme est très vacuolaire. La spore génératrice peut tomber assez tôt, laissant l'extrémité libre du champignon tronquée, ou persister longtemps. Les auteurs de langue française considéraient autrefois la spore génératrice comme un organe spécial, fertile ou stérile, caduc ou persistant; appelé «gland», ils lui attribuaient une réelle valeur systématique en faisant un critère spécifique, voire même générique (cf. Poisson, 1931 ; Manier, 1947 ; Duboscq, Léger et Tuzet, 1949).

Deux catégories de filaments se distinguent aisément: 1) des thalles de 7 à $12 \mu$ de large dans la partie médiane, renflés et recourbés dans la région proximale, fixés par un organe massif en forme de ventouse, ayant à peu près la largeur du filament dont il enchâsse la base et une hauteur de 5 à $7 \mu$ (fig. I, 3) ; 2) des thalles non coudés dans la partie proximale et dont le diamètre croît insensiblement de la base au sommet (ils ont par exemple à la base et au sommet : 6,6 et $10 \mu ; 7$ et $12 \mu ; 6,6$ et $8,8 \mu)$; ils sont attachés à l'intestin par de longs et grêles appendices qui s'élargissent seulement aux extrémités, d'un côté pour supporter le filament, de l'autre pour adhé-

(1) Leptaulax dentatus est un Passalide largement distribué dans la région orientale comprenant Bornéo et la Malaisie. Les échantillons nécessaires à cette étude ont été aimablement capturés par M. J. Rondon, que nous remercions vivement. Notre reconnaissance va également au Docteur C. Johnson, du Manchester Museum, qui a bien voulu se charger de la détermination de notre matériel. 


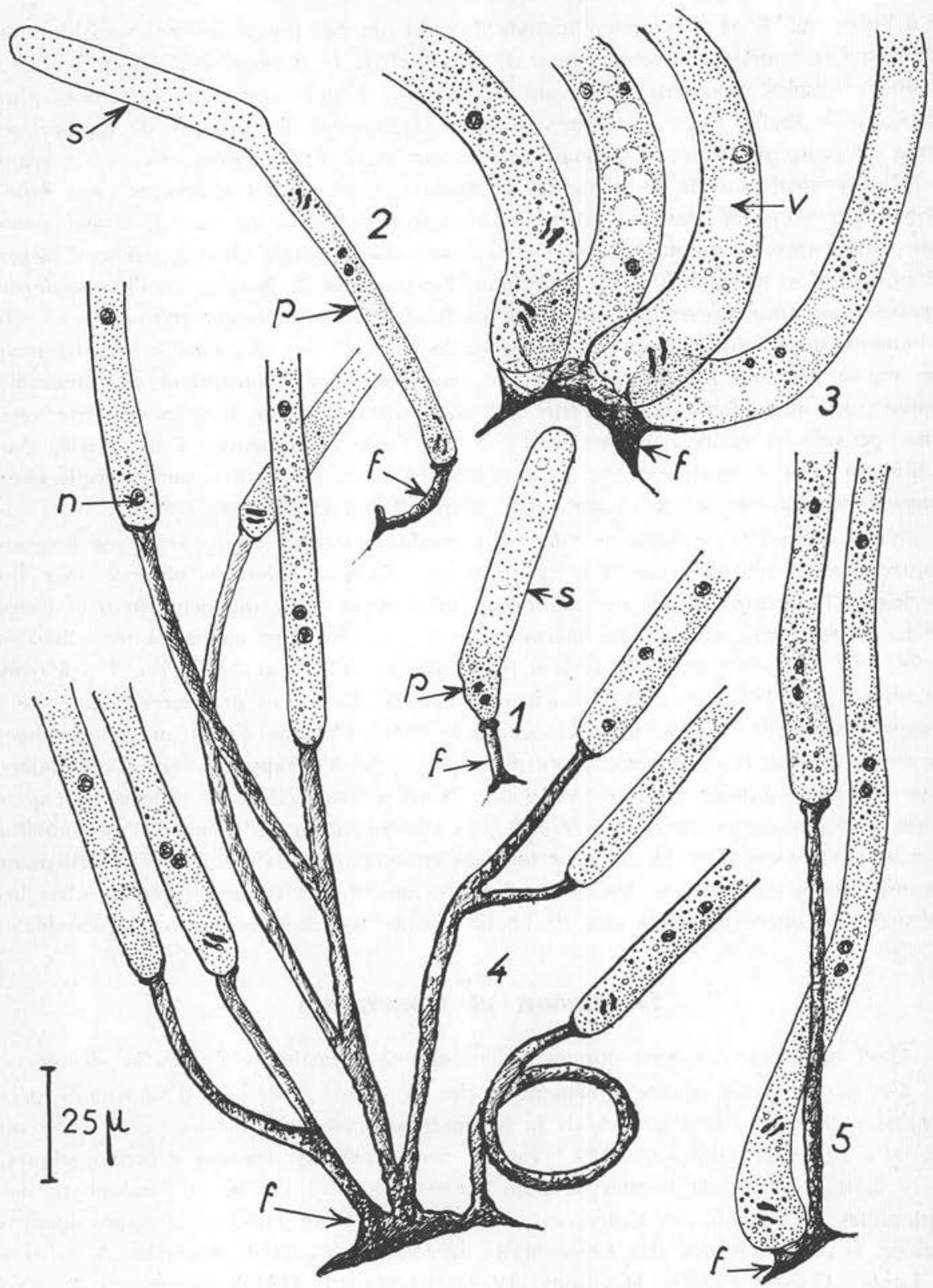

Fig. 1. - Enterobryus attenuatus : 1. germination d'une spore ; 2. jeune thalle ; 3 . régions basales d'un groupe de filaments à organe de fixation bas; 4. régions basales d'un groupe de filaments à longs organes de fixation; 5 . association d'un filament à organe de fixation en forme de ventouse et d'un filament à long appendice de fixation $(f$ : appendice de fixation; $n$ : noyau; $p$ : zone de prolifération; $s$ : spore; $v$ : vacuome) 
rer à l'hôte (fig. I, 4). Ces appendices de fixation ont des longueurs très variables, ils s'allongent au cours du développement du Thallophyte et peuvent avoir jusqu'à $170 \mu$ de long ; souples, onduleux, décrivant parfois une boucle complète, nettement plus grêles que le thalle, ils se distinguent toujours facilement des organes de fixation en forme de ventouse. Nous ne connaissons pas un autre Trichomycète Eccrinale ayant un tel polymorphisme de ses éléments de fixation. Leidy décrit seulement chez Enterobryus attenuatus de Passalus cornutus des organes de fixation courts, larges, coniques; Lichtwardt, reprenant l'étude d'E. attenuatus, signale des appendices larges $(17 \mu)$, ayant au plus de 50 à $70 \mu$ de haut. Les organes de fixation de l'Eccrinale de Leptaulax dentatus présentent une autre particularité; s'ils restent parfois isolés, ils fusionnent aussi fréquemment les uns avec les autres; les éléments courts forment une masse plus ou moins compacte, les éléments longs constituent un ensemble ramifié ; des appendices de longueurs très différentes, du type long et du type ventouse, peuvent se trouver assemblés (fig. I, 5). Cette coalescence, déjà signalée par Lichtwardt chez E. attenuatus, se retrouve chez d'autres Eccrinales, par exemple chez Enteromyces callianassae (cf. Lichtwardt, 1961; Tuzet et Manier, 1962).

Nous avons vu les filaments mûrs se reproduire presque uniquement par longues sporangiospores plurinucléées. Par ce processus classique, souvent observé chez les Eccrinales, l'extrémité distale des thalles se cloisonne et porte un, deux ou trois longs articles. Nous avons observé des filaments de $9 \mu$ de diamètre médian à trois articles de $98,150,125 \mu$ de long, de $12 \mu$ à deux articles de 235 et $230 \mu$, de $7 \mu$ à trois articles de $210,235,120 \mu$, de $10 \mu$ à un article de $75 \mu$. Les premières cellules formées sont susceptibles de se recloisonner; à maturité, chacune d'elles est un sporange, qui donne naissance à une seule spore plurinucléée qui s'échappe par une boutonnière et se fixe pour donner un nouveau thalle. Nous avons également observé quelques spores isodiamétriques, servant peut-être à la dissémination de l'espèce d'un individu à un autre. Comme c'est le cas pour tous les endophytes de Coléoptères actuellement connus, nous n'avons pas vu les spores durables qui se forment généralement chez les Eccrinales à l'approche de la mue de l'hôte et sont rejetées avec l'exuvie proctodéale.

\section{Discussion et Conclusion}

Quel nom pouvons-nous donner à l'endophyte parasite de Leptaulax dentatus ?

Ce parasite doit momentanément porter le nom générique d'Eccrinales déjà identifiées chez les Passalidae. Mais le nommerons-nous Enterobryus Leidy 1850 ou Trichella Léger et Duboscq 1929? En se basant sur les données morphologiques, Leidy était en droit de donner le nom d'Enterobryus à la fois à l'endophyte des Diplopodes et à celui des Coléoptères; en effet, à cette époque, la spore quadrinucléée, si caractéristique des Enterobryus de Diplopodes, était inconnue. A la suite de Leidy, Thaxter (1920), Heymons (1934), Lichtwardt (1957) conservent le nom d'Enterobryus pour les Eccrinales de Passalides. Par contre, Léger et Duboscq (1916), qui ne connaissaient pas davantage la spore quadrinucléée des Enterobryus, classent les Thallophytes de Coléoptères dans le genre Eccrinopsis, puis, en 1929, créent pour 
eux le genre Trichella. En l'absence d'observation de spores durables, ils justifient ainsi ce nouveau genre: "...Chez l'Eccrinide de l'Hydrophile, les microconides sont mises en liberté par un processus très particulier, élatérö̈de. De plus, dans un Hydrophilide, resté malheureusement indéterminé, nous avons vu des spores cylindriques à bouton latéral, qui semblaient bien provenir de l'Eccrinide observée en même temps qu'elles. » Léger et Duboscq ne se contentent pas d'établir le genre Trichella pour les parasites d'Hydrophilides, ils l'étendent, nous semble-t-il sans motif, aux endophytes de tous les Insectes et à ceux des Passalides en particulier. Depuis les travaux de Leidy et de Léger et Duboscq, les spores durables ovalaires quadrinucléées des Enterobryus de Diplopodes ont été à plusieurs reprises observées au moment de la mue de l'hôte (cf. Manier, 1950), mais elles n'ont jamais encore été vues chez les Passalides. Pour cette raison, serait-on en droit de créer deux genres, l'un pour les Thallophytes de Diplopodes, l'autre pour celui des Passalides? Un exemple pris parmi les Trichomycètes rameux nous invite à la prudence. Le genre Smittium fut établi en 1936 par Poisson pour un champignon filamenteux ramifié se reproduisant à la fois par spores asexuées et par spores sexuées; le genre Rubetella fut créé en 1961 par Tuzet, Rioux et Manier pour un Thallophyte comparable au précédent, mais chez lequel la reproduction sexuée ne se manifeste jamais; Lichtwardt (1964) conteste, pour nous à tort, la validité du genre Rubetella établi par une diagnose latine conformément aux règles internationales de la nomenclature botanique (Manier, 1963). Pour éviter des discussions stériles, il paraît plus raisonnable, pour le moment, de laisser l'endophyte du proctodeum de Leptaulax dentatus dans le genre Enterobryus (2). En ce qui concerne les noms spécifiques d'Eccrinales, jusqu'à ces dernières années, à un hôte nouveau correspondait presque toujours une espèce nouvelle. De plus, un endophyte, qui différait d'une forme connue par un seul caractère, était considéré comme nouveau. Ce processus a encombré la systématique d'espèces absolument injustifiables. Dans l'état actuel de nos connaissances, pour ne pas ajouter à la confusion de la classification des Eccrinales, nous rattacherons provisoirement, en insistant sur le caractère polymorphique de l'organe de fixation, l'Enterobryus de Leptaulax dentatus à l'espèce attenuatus de Leidy.

Actuellement, six genres d'Eccrinales ont été identifiés dans le proctodeum des Coléoptères. Ce sont : Enterobryus Leidy (1850), Trichella Léger et Duboscq (1929), Paratrichella Manier (1947), Lajassiella Tuzet et Manier (1950), Lactella Maessen (1955), Microtrichella Maessen (1955). Les critères des différents genres d'Eccrinales demeurent discutables. On tend aujourd'hui, pour clarifier la systématique, à supprimer de nombreux genres. Cependant, pour ne pas tomber dans l'excès contraire, nous conserverons trois genres: Enterobryus prioritaire, Trichella pour les Thallophytes d'Hydrophilides, Lajassiella (3) dont nous donnons la diagnose latine par celle de

(2) Le genre Enterobryus a été identifié dans des groupes d'Arthropodes très divers: Myriapodes, Diplopodes, Insectes Passalides, Crustacés Décapodes (Cronin et Johnson, 1958).

(3) Diagnosis Genus Lajassiella et species L. aphodii Tuzet et Manier (1950).

Eccrinales habentes filamenta simplicia modo siphonis. Quibus maturescentibus regerminatio sive spori globosis uninucleatis enascentibus ab articulis distalibus plurinucleatis; sive sporis ovatis uninucleatis enascentibus « in toto» thallo practer aliquam breviorem regionem proximalem. 
L. aphodii en note infrapaginale. Par contre, le genre Paratrichella, uniquement établi en raison de la présence d'un «gland», doit être supprimé et rapporté au genre Trichella; le genre Lactella, caractérisé seulement par ses filaments incolores de faible diamètre, est également à rayer des nomenclatures; le genre Microtrichella doit subir le sort que l'un de nous (Manier, 1961) a fait subir au genre Microeccrina, les grêles endophytes qui constituent ces genres étant des Schizophytes Arthromitaceae et non des Eccrinales.

Au cours de ces dernières années, la systématique des Trichomycètes Harpellales et Asellariales est devenue plus rigoureuse. Ces groupes, définitivement adoptés par les mycologues, ont été soumis aux règles internationales de nomenclature botanique. Certains de leurs représentants ont été cultivés in vitro (Whisler, 1960-1962 ; Clark, Kellen et Lindegren, 1963 ; Lichtwardt, 1964). Des expériences d'infestation croisée (Tuzet, Rioux et Manier, 1961 ; Manier, 1963), favorisées par la rapidité du cycle des hôtes (souvent larves aquatiques d'Insectes), ont prouvé que le même endophyte pouvait se développer dans des hôtes assez différents. Pour le moment, la biologie des Eccrinales est moins bien connue. Ces Thallophytes se cultivent mal ou ne se cultivent pas ; les hôtes ont souvent un cycle long et leur élevage en milieu stérile pose des problèmes. La systématique des Eccrinales se ressent de cette situation et demeure, dans bien des cas, provisoire, insatisfaisante.

\section{Résumé}

Après un historique concernant les Eccrinales d'Insectes Coléoptères, nous décrivons le Thallophyte parasite du proctodtum de Leptaulax dentatus L., Passalide provenant de la province de Vientiane au Laos. A l'issue d'une discussion systématique, nous nommons provisoirement ce parasite, dont les spores durables demeurent inconnues, Enterobryus attenuatus Leidy 1849. Nous maintenons, parmi les Eccrinales de Coléoptères, les genres Enterobryus, Trichella, Lajassiella, mais nous invalidons les genres Paratrichella, Lactella et Microtrichella.

\section{Summary}

After a short historical review on the Eccrinales parasitizing Coleoptera, the authors describe the Thallophyte inhabiting the proctodeum of Leptaulax dentatus L. a Passalid beetle from the area of Vientiane (Laos). After a taxonomical discussion, we classify temporarily this parasite, the lasting spores of which remain unknown, under the name Enterobryus attenuatus Leidy 1849. We maintain among the Eccrinales from Coleoptera the genera Enterobryus, Trichella, Lajassiella, but we invalidate the genera Paratrichella, Lactella and Microtrichella.

(Laboratoire de Zoologie de la Faculté des Sciences de Montpellier et Laboratoire d'évolution des êtres organisés de la Faculté des Sciences de Paris) 


\section{Bibliographie}

Clark (T. B.), Kellen (W. R.) et Lindegren (J. E.), 1963. - Axenic culture of two Trichomycetes from Californian. Mosquitoes. Nature, 197, $\mathrm{n}^{\circ}$ 4863, 208-209.

Cronin (E. T.) et Johnson (T. W.), 1958. - A halophilic Enterobryus in the mole Crab Emerita taepoïda Say. Journ. Elish. Mitch. Sc. Soc., 74, n 2, 167-172.

Dubosce (O.), LÉger (L.) et Tuzet (O.), 1948. Contribution à la connaissance des Eccrinides. Les Trichomycètes. Arch. Zool. Exp., 86, fasc. 2, 29-144.

Heymons (R. et H.), 1934. - Passalus und seine intestinale Flora. Biol. Zentralbl., 54, 40-51.

LÉGER (L.) et Dubosce (O.), 1934. - Les Eccrinides, nouveau groupe de protophytes parasites. C.R. Ac. Sc. Paris, 141, 425-427.

-, -, 1906. - L'évolution des Eccrina des Glomeris. C.R. Ac. Sc. Paris, 142, 590-592.

-, -, 1916. - Sur les Eccrinides des Hydrophilides. Arch. Zool. Exp., N. et R., n ${ }^{\circ}$ 2, 56, 21-31.

-, —, 1929. - Eccrinoides henneguyi n. g. et la systématique des Eccrinides. Arch. Anat. Microsc., $X X V, 309-324$.

LEIDY (J.), 1849. - a) Observations on species of Confervaceae on a new genus Enterobrus elegans, Cladophytum, a new genus of Entophyta, Cladophytum comatum, Arthromitus, a second genus. Proc. Acad. Sc., Phila, 4, 225-227.

-, 1849. - $-b$ ) Descriptions (accompanies by drawings) of new genera and species of Entophyta. Proc. Acad. Sc., Phila., 4, 249-250.

-, 1850. - Description of a new Entophyta growing within animals. Proc. Acad. Sc., Philadelphia, 5, 35-36.

-, 1853. - A Flora and Fauna within living animals. Smithsonian Contrib. to knowledge, 5, 1-67.

LichtWARDT (R. W.), 1957. - Enterobyrus attenuatus from the Passalid Beetle. Mycologia U.S.A., 49, $\mathrm{n}^{\circ} 4,463-474$.

-, 1962. — A stomach fungus in Callianassa spp (Decapoda) from Chile. Lunds Univ. Arsshr., 57, 3-10.

—, 1964. - Axenic culture of two new species of branched Trichomyctes. Journ. of Bot., $51, \mathrm{n}^{\circ} 8,836-842$.

MAessen (K), 1955. - Die Zooparasitaren Eccrinidales. Parasitologische Schriftenreihe, Heft 2, 129 pages.

Manier (J.-F.), 1947. - Paratrichella pentodoni n. g. n. sp., Entophyte parasite des larves de Pentodon punctatus De Vill. Ann. Sc. Nat. Zool., 11 $1^{\circ}$ série, 9, 275-279.

-, 1950. - Recherches sur les Trichomycètes. Ann. Sc. Nat. Bot., 11 série, 11, 54-162.

—, 1961. - Arthromitaceae, Schizophytes symbiotes de l'intestin postérieur des Myriapodes Diplopodes. Ann. Parasit. hum. et comparée, 36, $\mathrm{n}^{\circ}$ 1-2, 1-16.

—, 1963. — a) Trichomycètes parasites d'Isopodes Oniscoidea. Ann. Sc. Nat. Bot., $12^{\circ}$ sér., IV, fasc. 3, 557-578.

—, 1963. - b) Trichomycètes de larves de Simulies (Harpellales du proctodéum). Ann. Sc. Nat. Bot., $12^{\circ}$ série, 4 , fasc. 4, 737-750. 
— et THÉodoridès (L.), 1957. - Eccrinida d'un Gargilius sp. (Coléoptère Ténébrionide). Parc Nat. Albert, $2^{\circ}$ série, fasc. 5 (1), 3-6.

Poisson (R.), 1931. - Recherches sur les Eccrinides. Deuxième Contribution. Arch. Zool. Exp., 74, 53-68.

-, 1936. - Sur un endomycète nouveau: Smittium arvense n.g. n. sp., parasite intestinal des larves de Smittia sp. (Diptères Chironomides), et description d'une nouvelle espèce Stachylina Lég. et Gauth., 1932. Volume Jubilaire du $\mathrm{P}^{r}$ L. Daniel, Rennes, p. 1-11.

ThaXter (R.), 1920. - Second note on certain peculiar fungus parasites of living insects. Bot. Gazette, 69, 1-26.

THÉodoridès (J.), 1955. - Contribution à l'étude des parasites et phorétiques de Coléoptères terrestres. Act. Si. Ind. Hermann, n ${ }^{\circ}$ 1217, Suppl. n 4, «Vie et Milieu », 310 pages.

Tuzet (O.) et Manier (J.-F.), 1950. - Lajassiella aphodii n. g., n. sp. Palavascide parasite d'une larve d'Aphodius (Coléoptères Scarabaeidae). Ann. Sc. Nat., $11^{\circ}$ série, 12, 465-470.

-, -, 1962. - Enteromyces callianassae Lichwardt, Trichomycètes Eccrinale commencal de l'estomac de Uca pugilator. Latreille. Ann. Sc. Nat. Bot., $12^{\circ}$ série, 3, fasc. 4, 615-617.

-, Rioux (J.-A.) et MAnier (J.-F.), 1961. - Rubetella culicis (Tuzet et Manier, 1947), Trichomycète rameux parasite de l'ampoule rectale des larves de Culicides (morphologie et spécificité). Vie et Milieu, 12, 167-187.

Whisler (H.C.). - 1960. - Pure culture of the Trichomycete, Amoebidium parasiticum. Nature, $186, \mathrm{n}^{\circ} 4726,732-733$.

-, 1962. - Culture and nutrition of Amoebidium parasiticum. Amer. Journ. of Bot., 49, $\mathrm{n}^{\circ}$ 3, 193-199. 